Article

\title{
Third-Order Hankel and Toeplitz Determinants for Starlike Functions Connected with the Sine Function
}

\author{
Hai-Yan Zhang ${ }^{1}$ and Rekha Srivastava ${ }^{2, *}$ and Huo Tang ${ }^{1, *} \mathbb{1}$ \\ 1 School of Mathematics and Statistics, Chifeng University, Chifeng 024000, China; cfxyzhhy@163.com \\ 2 Department of Mathematics and Statistics, University of Victoria, Victoria, BC V8W 3R4, Canada \\ * Correspondence: rekhas@math.uvic.ca (R.S.); thth2009@163.com (H.T.)
}

Received: 26 March 2019; Accepted: 25 April 2019; Published: 6 May 2019

Abstract: Let $\mathcal{S}_{s}^{*}$ be the class of normalized functions $f$ defined in the open unit disk $\mathbb{D}=\{z:|z|<1\}$ such that the quantity $\frac{z f^{\prime}(z)}{f(z)}$ lies in an eight-shaped region in the right-half plane and satisfying the condition $\frac{z f^{\prime}(z)}{f(z)} \prec 1+\sin z(z \in \mathbb{D})$. In this paper, we aim to investigate the third-order Hankel determinant $H_{3}(1)$ and Toeplitz determinant $T_{3}(2)$ for this function class $\mathcal{S}_{S}^{*}$ associated with sine function and obtain the upper bounds of the determinants $H_{3}(1)$ and $T_{3}(2)$.

Keywords: starlike function; Toeplitz determinant; Hankel determinant; sine function; upper bound MSC: 30C45; 30C50; 30C 80

\section{Introduction} the form

Let $\mathcal{A}$ denote the class of functions $f$ which are analytic in the open unit disk $\mathbb{D}=\{z:|z|<1\}$ of

$$
f(z)=z+a_{2} z^{2}+a_{3} z^{3}+\cdots \quad(z \in \mathbb{D})
$$

and let $\mathcal{S}$ denote the subclass of $\mathcal{A}$ consisting of univalent functions.

Suppose that $\mathcal{P}$ denotes the class of analytic functions $p$ normalized by

$$
p(z)=1+c_{1} z+c_{2} z^{2}+c_{3} z^{3}+\cdots
$$

and satisfying the condition

$$
\Re(p(z))>0 \quad(z \in \mathbb{D}) .
$$

We easily see that, if $p(z) \in \mathcal{P}$, then a Schwarz function $\omega(z)$ exists with $\omega(0)=0$ and $|\omega(z)|<1$, such that (see [1])

$$
p(z)=\frac{1+w(z)}{1-w(z)} \quad(z \in \mathbb{D}) .
$$

Very recently, Cho et al. [2] introduced the following function class $\mathcal{S}_{s}^{*}$, which are associated with sine function:

$$
S_{s}^{*}:=\left\{f \in \mathcal{A}: \frac{z f^{\prime}(z)}{f(z)} \prec 1+\sin z(z \in \mathbb{D})\right\},
$$

where " $\prec$ " stands for the subordination symbol (for details, see [3]) and also implies that the quantity $\frac{z f^{\prime}(z)}{f(z)}$ lies in an eight-shaped region in the right-half plane. 
The $q^{\text {th }}$ Hankel determinant for $q \geq 1$ and $n \geq 1$ of functions $f$ was stated by Noonan and Thomas [4] as

$$
H_{q}(n)=\left|\begin{array}{cccc}
a_{n} & a_{n+1} & \cdots & a_{n+q-1} \\
& & & \\
a_{n+1} & a_{n+2} & \cdots & a_{n+q} \\
\vdots & \vdots & & \vdots \\
a_{n+q-1} & a_{n+q} & \cdots & a_{n+2 q-2}
\end{array}\right| \quad\left(a_{1}=1\right) .
$$

This determinant has been considered by several authors, for example, Noor [5] determined the rate of growth of $H_{q}(n)$ as $n \rightarrow \infty$ for functions $f(z)$ given by Equation (1) with bounded boundary and Ehrenborg [6] studied the Hankel determinant of exponential polynomials.

In particular, we have

$$
H_{3}(1)=\left|\begin{array}{lll}
a_{1} & a_{2} & a_{3} \\
a_{2} & a_{3} & a_{4} \\
a_{3} & a_{4} & a_{5}
\end{array}\right| \quad(n=1, q=3)
$$

Since $f \in \mathcal{S}, a_{1}=1$,

$$
H_{3}(1)=a_{3}\left(a_{2} a_{4}-a_{3}^{2}\right)-a_{4}\left(a_{4}-a_{2} a_{3}\right)+a_{5}\left(a_{3}-a_{2}^{2}\right) .
$$

We note that $\left|H_{2}(1)\right|=\left|a_{3}-a_{2}^{2}\right|$ is the well-known Fekete-Szego functional (see, for example, [7-9]).

On the other hand, Thomas and Halim [10] defined the symmetric Toeplitz determinant $T_{q}(n)$ as follows:

$$
T_{q}(n)=\left|\begin{array}{cccc}
a_{n} & a_{n+1} & \cdots & a_{n+q-1} \\
a_{n+1} & a_{n} & \cdots & a_{n+q} \\
\vdots & \vdots & & \vdots \\
a_{n+q-1} & a_{n+q} & \cdots & a_{n}
\end{array}\right| \quad(n \geq 1, q \geq 1) .
$$

The Toeplitz determinants are closely related to Hankel determinants. Hankel matrices have constant entries along the reverse diagonal, whereas Toeplitz matrices have constant entries along the diagonal. For a good summary of the applications of Toeplitz matrices to the wide range of areas of pure and applied mathematics, we can refer to [11].

As a special case, when $n=2$ and $q=3$, we have

$$
T_{3}(2)=\left|\begin{array}{lll}
a_{2} & a_{3} & a_{4} \\
a_{3} & a_{2} & a_{3} \\
a_{4} & a_{3} & a_{2}
\end{array}\right| .
$$

In recent years, many authors studied the second-order Hankel determinant $H_{2}(2)$ and the third-order Hankel determinant $H_{3}(1)$ for various classes of functions (the interested readers can see, for instance, [12-25]). However, apart from the work in $[10,21,26,27]$, there appears to be little literature dealing with Toeplitz determinants. Inspired by the aforementioned works, in this paper, we aim to investigate the third-order Hankel determinant $H_{3}(1)$ and Toeplitz determinant $T_{3}(2)$ for the above function class $\mathcal{S}_{\mathcal{S}}^{*}$ associated with sine function, and obtain the upper bounds of the above determinants. 


\section{Main Results}

To prove our desired results, we need the following lemmas.

Lemma 1. If $p(z) \in \mathcal{P}$, then exists some $x, z$ with $|x| \leq 1$ (see [28]), $|z| \leq 1$, such that

$$
\begin{gathered}
2 c_{2}=c_{1}^{2}+x\left(4-c_{1}^{2}\right) \\
4 c_{3}=c_{1}^{3}+2 c_{1} x\left(4-c_{1}^{2}\right)-\left(4-c_{1}^{2}\right) c_{1} x^{2}+2\left(4-c_{1}^{2}\right)\left(1-|x|^{2}\right) z .
\end{gathered}
$$

Lemma 2. Let $p(z) \in \mathcal{P}($ see [29]), then

$$
\left|c_{n}\right| \leq 2, n=1,2, \cdots .
$$

We now state and prove the main results of our present investigation.

Theorem 1. If the function $f(z) \in \mathcal{S}_{s}^{*}$ and of the form Equation (1), then

$$
\left|a_{2}\right| \leq 1,\left|a_{3}\right| \leq \frac{1}{2},\left|a_{4}\right| \leq \frac{5}{9},\left|a_{5}\right| \leq \frac{47}{72} .
$$

Proof. Since $f(z) \in \mathcal{S}_{S}^{*}$, according to subordination relationship, so there exists a Schwarz function $\omega(z)$ with $\omega(0)=0$ and $|\omega(z)|<1$, such that

$$
\frac{z f^{\prime}(z)}{f(z)}=1+\sin (\omega(z))
$$

Now,

$$
\begin{gathered}
\frac{z f^{\prime}(z)}{f(z)}=\frac{z+\sum_{n=2}^{\infty} n a_{n} z^{n}}{z+\sum_{n=2}^{\infty} a_{n} z^{n}} \\
=\left(1+\sum_{n=2}^{\infty} n a_{n} z^{n-1}\right)\left[1-a_{2} z+\left(a_{2}^{2}-a_{3}\right) z^{2}-\left(a_{2}^{3}-2 a_{2} a_{3}+a_{4}\right) z^{3}\right. \\
\left.+\left(a_{2}^{4}-3 a_{2}^{2} a_{3}+2 a_{2} a_{4}+a_{3}^{2}-a_{5}\right) z^{4}+\cdots\right] \\
=1+a_{2} z+\left(2 a_{3}-a_{2}^{2}\right) z^{2}+\left(a_{2}^{3}-3 a_{2} a_{3}+3 a_{4}\right) z^{3} \\
+\left(4 a_{5}-a_{2}^{4}+4 a_{2}^{2} a_{3}-4 a_{2} a_{4}-2 a_{3}^{2}\right) z^{4}+\cdots .
\end{gathered}
$$

Define a function

$$
p(z)=\frac{1+\omega(z)}{1-\omega(z)}=1+c_{1} z+c_{2} z^{2}+\cdots .
$$

Clearly, we have $p(z) \in \mathcal{P}$ and

$$
\omega(z)=\frac{p(z)-1}{1+p(z)}=\frac{c_{1} z+c_{2} z^{2}+c_{3} z^{3}+\cdots}{2+c_{1} z+c_{2} z^{2}+c_{3} z^{3}+\cdots} .
$$

On the other hand,

$$
\begin{gathered}
1+\sin (\omega(z))=1+\frac{1}{2} c_{1} z+\left(\frac{c_{2}}{2}-\frac{c_{1}^{2}}{4}\right) z^{2}+\left(\frac{5 c_{1}^{3}}{48}+\frac{c_{3}-c_{1} c_{2}}{2}\right) z^{3} \\
+\left(\frac{c_{4}}{2}+\frac{5 c_{1}^{2} c_{2}}{16}-\frac{c_{2}^{2}}{4}-\frac{c_{1} c_{3}}{2}-\frac{c_{1}^{4}}{32}\right) z^{4}+\cdots .
\end{gathered}
$$


Comparing the coefficients of $z, z^{2}, z^{3}, z^{4}$ between Equations (4) and (6), we obtain

$$
a_{2}=\frac{c_{1}}{2}, a_{3}=\frac{c_{2}}{4}, a_{4}=\frac{c_{3}}{6}-\frac{c_{1} c_{2}}{24}-\frac{c_{1}^{3}}{144}, a_{5}=\frac{c_{4}}{8}-\frac{c_{1} c_{3}}{24}+\frac{5 c_{1}^{4}}{1152}-\frac{c_{1}^{2} c_{2}}{192}-\frac{c_{2}^{2}}{32} .
$$

By using Lemma 2, we thus know that

$$
\left|a_{2}\right| \leq 1,\left|a_{3}\right| \leq \frac{1}{2},\left|a_{4}\right| \leq \frac{5}{9},\left|a_{5}\right| \leq \frac{47}{72} .
$$

The proof of Theorem 1 is completed.

Theorem 2. If the function $f(z) \in \mathcal{S}_{s}^{*}$ and of the form in Equation (1), then we have

$$
\left|a_{3}-a_{2}^{2}\right| \leq \frac{1}{2}
$$

Proof. According to Equation (7), we have

$$
\left|a_{3}-a_{2}^{2}\right|=\left|\frac{c_{2}}{4}-\frac{c_{1}^{2}}{4}\right| .
$$

By applying Lemma 1, we get

$$
\left|a_{3}-a_{2}^{2}\right|=\left|\frac{x\left(4-c_{1}^{2}\right)}{8}-\frac{c_{1}^{2}}{8}\right| .
$$

Let $|x|=t, t \in[0,1], c_{1}=c, c \in[0,2]$. Then, using the triangle inequality, we obtain

$$
\left|a_{3}-a_{2}^{2}\right| \leq \frac{t\left(4-c^{2}\right)}{8}+\frac{c^{2}}{8} .
$$

Suppose that

$$
F(c, t)=\frac{t\left(4-c^{2}\right)}{8}+\frac{c^{2}}{8}
$$

then $\forall t \in(0,1), \forall c \in(0,2)$,

$$
\frac{\partial F}{\partial t}=\frac{4-c^{2}}{8}>0,
$$

which shows that $F(c, t)$ is an increasing function on the closed interval $[0,1]$ about $t$. Therefore, the function $F(c, t)$ can get the maximum value at $t=1$, that is, that

$$
\max F(c, t)=F(c, 1)=\frac{\left(4-c^{2}\right)}{8}+\frac{c^{2}}{8}=\frac{1}{2} .
$$

Thus, obviously,

$$
\left|a_{3}-a_{2}^{2}\right| \leq \frac{1}{2}
$$

The proof of Theorem 2 is thus completed.

Theorem 3. If the function $f(z) \in \mathcal{S}_{\mathcal{S}}^{*}$ and of the form in Equation (1), then we have

$$
\left|a_{2} a_{3}-a_{4}\right| \leq \frac{1}{3}
$$


Proof. From Equation (7), we have

$$
\begin{aligned}
\left|a_{2} a_{3}-a_{4}\right| & =\left|\frac{c_{1} c_{2}}{8}+\frac{c_{1}^{3}}{144}-\frac{c_{3}}{6}+\frac{c_{1} c_{2}}{24}\right| \\
= & \left|\frac{c_{1} c_{2}}{6}-\frac{c_{3}}{6}+\frac{c_{1}^{3}}{144}\right| .
\end{aligned}
$$

Now, in view of Lemma 1, we get

$$
\left|a_{2} a_{3}-a_{4}\right|=\left|\frac{7 c_{1}^{3}}{144}+\frac{\left(4-c_{1}^{2}\right) c_{1} x^{2}}{24}-\frac{\left(4-c_{1}^{2}\right)\left(1-|x|^{2}\right) z}{12}\right| .
$$

Let $|x|=t, t \in[0,1], c_{1}=c, c \in[0,2]$. Then, using the triangle inequality, we deduce that

$$
\left|a_{2} a_{3}-a_{4}\right| \leq \frac{7 c^{3}}{144}+\frac{\left(4-c^{2}\right) c t^{2}}{24}+\frac{\left(4-c^{2}\right)\left(1-t^{2}\right)}{12} .
$$

Assume that

$$
F(c, t)=\frac{7 c^{3}}{144}+\frac{\left(4-c^{2}\right) c t^{2}}{24}+\frac{\left(4-c^{2}\right)\left(1-t^{2}\right)}{12} .
$$

Therefore, we have, $\forall t \in(0,1), \forall c \in(0,2)$

$$
\frac{\partial F}{\partial t}=\frac{\left(4-c^{2}\right) t(c-2)}{12}<0,
$$

namely, $F(c, t)$ is an decreasing function on the closed interval $[0,1]$ about $t$. This implies that the maximum value of $F(c, t)$ occurs at $t=0$, which is

$$
\max F(c, t)=F(c, 0)=\frac{\left(4-c^{2}\right)}{12}+\frac{7 c^{3}}{144} \text {. }
$$

Define

$$
G(c)=\frac{\left(4-c^{2}\right)}{12}+\frac{7 c^{3}}{144}
$$

clearly, the function $G(c)$ has a maximum value attained at $c=0$, also which is

$$
\left|a_{2} a_{3}-a_{4}\right| \leq G(0)=\frac{1}{3} .
$$

The proof of Theorem 3 is completed.

Theorem 4. If the function $f(z) \in \mathcal{S}_{S}^{*}$ and of the form in Equation (1), then we have

$$
\left|a_{2} a_{4}-a_{3}^{2}\right| \leq \frac{1}{4}
$$

Proof. Suppose that $f(z) \in \mathcal{S}_{s}^{*}$, then from Equation (7), we have

$$
\left|a_{2} a_{4}-a_{3}^{2}\right|=\left|\frac{c_{1} c_{3}}{12}-\frac{c_{1}^{2} c_{2}}{48}+\frac{c_{1}^{4}}{48}-\frac{c_{2}^{2}}{16}\right| .
$$

Now, in terms of Lemma 1, we obtain

$$
\begin{aligned}
\left|a_{2} a_{4}-a_{3}^{2}\right| & =\left|\frac{c_{1} c_{3}}{12}-\frac{c_{1}^{2} c_{2}}{48}-\frac{c_{1}^{4}}{288}-\frac{c_{2}^{2}}{16}\right| \\
= & \left|-\frac{5 c_{1}^{4}}{576}-\frac{x^{2} c_{1}^{2}\left(4-c_{1}^{2}\right)}{48}-\frac{x^{2}\left(4-c_{1}^{2}\right)^{2}}{64}+\frac{c_{1}\left(4-c_{1}^{2}\right)\left(1-|x|^{2}\right) z}{24}\right| .
\end{aligned}
$$


Let $|x|=t, t \in[0,1], c_{1}=c, c \in[0,2]$. Then, using the triangle inequality, we get

$$
\left|a_{2} a_{4}-a_{3}^{2}\right| \leq \frac{t^{2} c^{2}\left(4-c^{2}\right)}{48}+\frac{\left(1-t^{2}\right) c\left(4-c^{2}\right)}{24}+\frac{t^{2}\left(4-c^{2}\right)^{2}}{64}+\frac{5 c^{4}}{576} .
$$

Putting

$$
F(c, t)=\frac{t^{2} c^{2}\left(4-c^{2}\right)}{48}+\frac{\left(1-t^{2}\right) c\left(4-c^{2}\right)}{24}+\frac{t^{2}\left(4-c^{2}\right)^{2}}{64}+\frac{5 c^{4}}{576},
$$

then, $\forall t \in(0,1), \forall c \in(0,2)$, we have

$$
\frac{\partial F}{\partial t}=\frac{t\left(c^{2}-8 c+12\right)\left(4-c^{2}\right)}{96}>0
$$

which implies that $F(c, t)$ increases on the closed interval $[0,1]$ about $t$. That is, that $F(c, t)$ have a maximum value at $t=1$, which is

$$
\max F(c, t)=F(c, 1)=\frac{c^{2}\left(4-c^{2}\right)}{48}+\frac{\left(4-c^{2}\right)^{2}}{64}+\frac{5 c^{4}}{576} .
$$

Setting

$$
G(c)=\frac{c^{2}\left(4-c^{2}\right)}{48}+\frac{\left(4-c^{2}\right)^{2}}{64}+\frac{5 c^{4}}{576}
$$

then we have

$$
G^{\prime}(c)=\frac{c\left(4-c^{2}\right)}{24}-\frac{c^{3}}{24}-\frac{c\left(4-c^{2}\right)}{16}+\frac{5 c^{3}}{144} .
$$

If $G^{\prime}(c)=0$, then the root is $c=0$. In addition, since $G^{\prime \prime}(0)=-\frac{1}{12}<0$, so the function $G(c)$ can take the maximum value at $c=0$, which is

$$
\left|a_{2} a_{4}-a_{3}^{2}\right| \leq G(0)=\frac{1}{4} .
$$

The proof of Theorem 4 is completed.

Theorem 5. If the function $f(z) \in \mathcal{S}_{\mathcal{S}}^{*}$ and of the form in Equation (1), then we have

$$
\left|a_{2}^{2}-a_{3}^{2}\right| \leq \frac{5}{4}
$$

Proof. Suppose that $f(z) \in \mathcal{S}_{s}^{*}$, then, by using Equation (7), we have

$$
\left|a_{2}^{2}-a_{3}^{2}\right|=\left|\frac{c_{1}^{2}}{4}-\frac{c_{2}^{2}}{16}\right| .
$$

Next, according to Lemma 1, we obtain

$$
\begin{aligned}
& \left|a_{2}^{2}-a_{3}^{2}\right|=\left|\frac{c_{1}^{2}}{4}-\frac{c_{2}^{2}}{16}\right| \\
& =\left|\frac{c_{1}^{2}}{4}-\frac{c_{1}^{4}}{64}-\frac{x c_{1}^{2}\left(4-c_{1}^{2}\right)}{32}-\frac{x^{2}\left(4-c_{1}^{2}\right)^{2}}{64}\right| .
\end{aligned}
$$

Let $|x|=t, t \in[0,1], c_{1}=c, c \in[0,2]$. Then, by applying the triangle inequality, we get

$$
\left|a_{2}^{2}-a_{3}^{2}\right| \leq \frac{c^{2}}{4}+\frac{c^{4}}{64}+\frac{t c^{2}\left(4-c^{2}\right)}{32}+\frac{t^{2}\left(4-c^{2}\right)^{2}}{64} .
$$


Taking

$$
F(c, t)=\frac{c^{2}}{4}+\frac{c^{4}}{64}+\frac{t c^{2}\left(4-c^{2}\right)}{32}+\frac{t^{2}\left(4-c^{2}\right)^{2}}{64} .
$$

Then, $\forall t \in(0,1), \forall c \in(0,2)$, we have

$$
\frac{\partial F}{\partial t}=\frac{c^{2}\left(4-c^{2}\right)}{32}+\frac{t\left(4-c^{2}\right)^{2}}{32}>0,
$$

which implies that $F(c, t)$ increases on the closed interval [0,1] about $t$. Namely, the maximum value of $F(c, t)$ attains at $t=1$, which is

$$
\max F(c, t)=F(c, 1)=\frac{c^{2}}{4}+\frac{c^{4}}{64}+\frac{c^{2}\left(4-c^{2}\right)}{32}+\frac{\left(4-c^{2}\right)^{2}}{64} .
$$

Let

$$
G(c)=\frac{c^{2}}{4}+\frac{c^{4}}{64}+\frac{c^{2}\left(4-c^{2}\right)}{32}+\frac{\left(4-c^{2}\right)^{2}}{64}
$$

then

$$
G^{\prime}(c)=\frac{c}{2}>0, \forall c \in(0,2) .
$$

Therefore, the function $G(c)$ is an increasing function on the closed interval $[0,2]$ about $c$, and thus $G(c)$ has a maximum value attained at $c=2$, which is

$$
\left|a_{2}^{2}-a_{3}^{2}\right| \leq G(2)=\frac{5}{4}
$$

The proof of Theorem 5 is completed.

Theorem 6. If the function $f(z) \in \mathcal{S}_{S}^{*}$ and of the form in Equation (1), then we have

$$
\left|a_{2} a_{3}-a_{3} a_{4}\right| \leq \frac{13}{12}
$$

Proof. Assume that $f(z) \in \mathcal{S}_{S}^{*}$, then from Equation (7), we obtain

$$
\left|a_{2} a_{3}-a_{3} a_{4}\right|=\left|\frac{c_{1} c_{2}}{8}+\frac{c_{1}^{3} c_{2}}{576}-\frac{c_{2} c_{3}}{24}+\frac{c_{1} c_{2}^{2}}{96}\right| .
$$

Now, by using Lemma 1, we see that

$$
\begin{aligned}
& \left|a_{2} a_{3}-a_{3} a_{4}\right|=\left|\frac{c_{1} c_{2}}{8}+\frac{c_{1}^{3} c_{2}}{576}-\frac{c_{2} c_{3}}{24}+\frac{c_{1} c_{2}^{2}}{96}\right| \\
& \quad=\left|\frac{c_{1}^{3}}{16}-\frac{c_{1}^{5}}{576}-\frac{11 x c_{1}^{3}\left(4-c_{1}^{2}\right)}{1152}+\frac{x c_{1}\left(4-c_{1}^{2}\right)}{16}+\frac{x^{2} c_{1}\left(4-c_{1}^{2}\right)\left[c_{1}^{2}+x\left(4-c_{1}^{2}\right)\right]}{192}+\frac{c_{1} x^{2}\left(4-c_{1}^{2}\right)^{2}}{128}+\frac{\left(1-|x|^{2}\right) z\left(4-c_{1}^{2}\right)\left[x\left(4-c_{1}^{2}\right)+c_{1}^{2}\right]}{96}\right| .
\end{aligned}
$$

If we let $|x|=t, t \in[0,1], c_{1}=c, c \in[0,2]$, then, using the triangle inequality, we have

$$
\left|a_{2} a_{3}-a_{3} a_{4}\right| \leq \frac{c^{3}}{16}+\frac{c^{5}}{576}+\frac{11 t c^{3}\left(4-c^{2}\right)}{1152}+\frac{t\left(4-c^{2}\right)}{8}+\frac{t^{2}\left[c^{2}+t\left(4-c^{2}\right)\right]\left(4-c^{2}\right)}{96}+\frac{t^{2}\left(4-c^{2}\right)^{2}}{64}+\frac{\left(4-c^{2}\right)\left[t\left(4-c^{2}\right)+c^{2}\right]}{96}
$$

Setting

$$
F(c, t)=\frac{c^{3}}{16}+\frac{c^{5}}{576}+\frac{11 t c^{3}\left(4-c^{2}\right)}{1152}+\frac{t\left(4-c^{2}\right)}{8}+\frac{t^{2}\left[c^{2}+t\left(4-c^{2}\right)\right]\left(4-c^{2}\right)}{96}+\frac{t^{2}\left(4-c^{2}\right)^{2}}{64}+\frac{\left(4-c^{2}\right)\left[t\left(4-c^{2}\right)+c^{2}\right]}{96} .
$$

Then, we easily see that, $\forall t \in(0,1), \forall c \in(0,2)$,

$$
\frac{\partial F}{\partial t}=\frac{11 c^{3}\left(4-c^{2}\right)}{1152}+\frac{\left(4-c^{2}\right)}{8}+\frac{t\left[c^{2}+t\left(4-c^{2}\right)\right]\left(4-c^{2}\right)}{48}+\frac{t^{2}\left(4-c^{2}\right)^{2}}{96}+\frac{t\left(4-c^{2}\right)^{2}}{32}+\frac{\left(4-c^{2}\right)^{2}}{96}>0,
$$


which implies that $F(c, t)$ is an increasing function on the closed interval $[0,1]$ about $t$. That is, that the maximum value of $F(c, t)$ occurs at $t=1$, which is

$$
\max F(c, t)=F(c, 1)=\frac{c^{3}}{16}+\frac{c^{5}}{576}+\frac{11 c^{3}\left(4-c^{2}\right)}{1152}+\frac{\left(4-c^{2}\right)}{8}+\frac{\left(4-c^{2}\right)}{24}+\frac{\left(4-c^{2}\right)^{2}}{64}+\frac{\left(4-c^{2}\right)}{24} .
$$

Taking

$$
G(c)=\frac{c^{3}}{16}+\frac{c^{5}}{576}+\frac{11 c^{3}\left(4-c^{2}\right)}{1152}+\frac{\left(4-c^{2}\right)}{8}+\frac{\left(4-c^{2}\right)}{24}+\frac{\left(4-c^{2}\right)^{2}}{64}+\frac{\left(4-c^{2}\right)}{24},
$$

then

$$
\begin{aligned}
& G^{\prime}(c)=\frac{3 c^{2}}{16}+\frac{5 c^{4}}{576}+\frac{11 c^{2}\left(4-c^{2}\right)}{384}-\frac{11 c^{4}}{576}-\frac{c\left(4-c^{2}\right)}{16}-\frac{c}{12} \\
& G^{\prime \prime}(c)=\frac{3 c}{8}+\frac{5 c^{3}}{144}+\frac{11 c\left(4-2 c^{2}\right)}{192}-\frac{11 c^{3}}{144}-\frac{\left(4-c^{2}\right)}{16}+\frac{c^{2}}{8}-\frac{1}{12}
\end{aligned}
$$

We easily find that $c=0$ is the root of the function $G^{\prime}(c)=0$, since $G^{\prime \prime}(0)<0$, which implies that the function $G(c)$ can reach the maximum value at $c=0$, also which is

$$
\left|a_{2} a_{3}-a_{3} a_{4}\right| \leq G(0)=\frac{13}{12} .
$$

The proof of Theorem 6 is completed.

Theorem 7. If the function $f(z) \in \mathcal{S}_{\mathcal{S}}^{*}$ and of the form in Equation (1), then we have

$$
\left|H_{3}(1)\right| \leq \frac{275}{432} \approx 0.637 \text {. }
$$

Proof. Since

$$
H_{3}(1)=a_{3}\left(a_{2} a_{4}-a_{3}^{2}\right)-a_{4}\left(a_{4}-a_{2} a_{3}\right)+a_{5}\left(a_{3}-a_{2}^{2}\right),
$$

by applying the triangle inequality, we get

$$
\left|H_{3}(1)\right| \leq\left|a_{3}\right|\left|a_{2} a_{4}-a_{3}^{2}\right|+\left|a_{4}\right|\left|a_{4}-a_{2} a_{3}\right|+\left|a_{5}\right|\left|a_{3}-a_{2}^{2}\right| .
$$

Now, substituting Equations (3), (8), (9) and (10) into Equation (14), we easily obtain the desired assertion (Equation (13)).

Theorem 8. If the function $f(z) \in \mathcal{S}_{\mathcal{S}}^{*}$ and of the form in Equation (1), then we have

$$
\left|T_{3}(2)\right| \leq \frac{139}{72} \approx 1.931 .
$$

Proof. Because

$$
T_{3}(2)=a_{2}\left(a_{2}^{2}-a_{3}^{2}\right)-a_{3}\left(a_{2} a_{3}-a_{3} a_{4}\right)+a_{4}\left(a_{3}^{2}-a_{2} a_{4}\right),
$$

by using the triangle inequality, we obtain

$$
\left|T_{3}(2)\right| \leq\left|a_{2}\right|\left|a_{2}^{2}-a_{3}^{2}\right|+\left|a_{3}\right|\left|a_{2} a_{3}-a_{3} a_{4}\right|+\left|a_{4}\right|\left|a_{3}^{2}-a_{2} a_{4}\right| .
$$

Next, from Equations (3), (10), (11) and (12), we immediately get the desired assertion (Equation (15)).

Finally, we give two examples to illustrate our results obtained. 
Example 1. If we take the function $f(z)=e^{z}-1=z+\sum_{n=2}^{\infty} \frac{z^{n}}{n !} \in \mathcal{S}_{S}^{*}$, then we obtain

$$
\begin{aligned}
& \left|H_{3}(1)\right| \leq\left|a_{3}\right|\left|a_{2} a_{4}-a_{3}^{2}\right|+\left|a_{4}\right|\left|a_{4}-a_{2} a_{3}\right|+\left|a_{5}\right|\left|a_{3}-a_{2}^{2}\right| \\
& =\frac{1}{3 !} \times\left|\frac{1}{2 !} \times \frac{1}{4 !}-\frac{1}{3 !} \times \frac{1}{3 !}\right|+\frac{1}{4 !} \times\left|\frac{1}{4 !}-\frac{1}{2 !} \times \frac{1}{3 !}\right|+\frac{1}{5 !} \times\left|\frac{1}{3 !}-\frac{1}{2 !} \times \frac{1}{2 !}\right| \\
& \quad \approx 0.004<0.637 .
\end{aligned}
$$

Example 2. If we set the function $f(z)=-\log (1-z)=z+\sum_{n=2}^{\infty} \frac{z^{n}}{n} \in \mathcal{S}_{s}^{*}$, then we get

$$
\begin{aligned}
& \left|T_{3}(2)\right| \leq\left|a_{2}\right|\left|a_{2}^{2}-a_{3}^{2}\right|+\left|a_{3}\right|\left|a_{2} a_{3}-a_{3} a_{4}\right|+\left|a_{4}\right|\left|a_{3}^{2}-a_{2} a_{4}\right| \\
& =\frac{1}{2} \times\left|\frac{1}{2} \times \frac{1}{2}-\frac{1}{3} \times \frac{1}{3}\right|+\frac{1}{3} \times\left|\frac{1}{2} \times \frac{1}{3}-\frac{1}{3} \times \frac{1}{4}\right|+\frac{1}{4} \times\left|\frac{1}{3} \times \frac{1}{3}-\frac{1}{2} \times \frac{1}{4}\right| \\
& \quad \approx 0.107<1.931 .
\end{aligned}
$$

Author Contributions: conceptualization, H.T. and H.-Y.Z.; methodology, H.T. and H.-Y.Z.; software, H.-Y.Z.; validation, H.-Y.Z, R.S. and H.T.; formal analysis, R.S.; investigation, H.T.; resources, H.T.; data curation, H.T.; writing-original draft preparation, H.-Y.Z.; writing-review and editing, H.T. and R.S.; visualization, R.S.; supervision, H.T. and R.S.; project administration, H.T.; funding acquisition, H.T.

Funding: This research was funded by the Natural Science Foundation of the People's Republic of China under Grants 11561001 and 11271045, the Program for Young Talents of Science and Technology in Universities of Inner Mongolia Autonomous Region under Grant NJYT-18-A14, the Natural Science Foundation of Inner Mongolia of the People's Republic of China under Grant 2018MS01026, the Higher School Foundation of Inner Mongolia of the People's Republic of China under Grants NJZY17300 and NJZY18217 and the Natural Science Foundation of Chifeng of Inner Mongolia.

Conflicts of Interest: The authors declare no conflict of interest.

\section{References}

1. Srivastava, H.M.; Owa, S. Current Topics in Analytic Function Theory; World Scientific Publishing Company: Singapore, 1992.

2. Cho, N.E.; Kumar, V.; Kumar, S.S.; Ravichandran, V. Radius problems for starlike functions associated with the Sine function. Bull. Iran. Math. Soc. 2019, 45, 213-232. [CrossRef]

3. Miller, S.S.; Mocanu, P.T. Differential Subordinations: Theory and Applications, Series on Monographs and Textbooks in Pure and Applied Mathematics, No. 225; Marcel Dekker Incorporated: New York, NY, USA, 2000.

4. Noonan, J.W.; Thomas, D.K. On the second Hankel determinant of areally mean $p$-valent functions. Trans. Am. Math. Soc. 1976, 223, 337-346.

5. Noor, K.I. Hankel determinant problem for the class of functions with bounded boundary rotation. Rev. Roumaine Math. Pure Appl. 1983, 28, 731-739.

6. Ehrenborg, R. The Hankel determinant of exponential polynomials. Am. Math. Mon. 2000, 107, 557-560. [CrossRef]

7. Fekete, M.; Szegö, G. Eine benberkung uber ungerada schlichte funktionen. J. Lond. Math. Soc. 1933, 8, 85-89. [CrossRef]

8. Koepf, W. On the Fekete-Szego problem for close-to-convex functions. Proc. Am. Math. Soc. 1987, 101, 89-95.

9. Koepf, W. On the Fekete-Szego problem for close-to-convex functions II. Arch. Math. 1987, 49, $420-433$. [CrossRef]

10. Thomas, D.K.; Halim, S.A. Toeplitz matrices whose elements are the coefficients of starlike and close-to-convex functions. Bull. Malays. Math. Sci. Soc. 2017. [CrossRef]

11. Ye, K.; Lim, L.-H. Every matrix is a product of Toeplitz matrices. Found. Comput. Math. 2016, 16, 577-598. [CrossRef]

12. Babalola, K.O. On $\mathrm{H}_{3}(1)$ Hankel determinant for some classes of univalent functions. Inequal. Theory Appl. 2010, 6, 1-7. 
13. Bansal, D. Upper bound of second Hankel determinant for a new class of analytic functions. Appl. Math. Lett. 2013, 26, 103-107. [CrossRef]

14. Bansal, D.; Maharana, S.; Prajapat, J.K. Third order Hankel determinant for certain univalent functions. J. Korean Math. Soc. 2015, 52, 1139-1148. [CrossRef]

15. Caglar, M.; Deniz, E.; Srivastava, H.M. Second Hankel determinant for certain subclasses of bi-univalent functions. Turk. J. Math. 2017, 41, 694-706. [CrossRef]

16. Janteng, A.; Halim, S.; Darus, M. Coefficient inequality for a function whose derivative has a positive real part. J. Inequal. Pure Appl. Math. 2006, 50, 5.

17. Janteng, A.; Halim, S.A.; Darus, M. Hankel determinant for starlike and convex functions. Int. J. Math. Anal. 2007, 13, 619-625.

18. Lee, S.K.; Ravichandran, V.; Subramaniam, S. Bounds for the second Hankel determinant of certain univalent functions. J. Inequal. Appl. 2013, 281, 17. [CrossRef]

19. Mahmood, S.; Srivastava, H.M.; Khan, N.; Ahmad, Q.Z.; Khan, B.; Ali, I. Upper bound of the third Hankel determinant for a subclass of $q$-starlike functions. Symmetry 2019, 11, 1-13. [CrossRef]

20. Raza, M.; Malik, S.N. Upper bound of the third Hankel determinant for a class of analytic functions related with lemniscate of Bernoulli. J. Inequal. Appl. 2013, 2013, 1-8. [CrossRef]

21. Srivastava, H.M.; Ahmad, Q.Z.; Khan, N.; Khan, B. Hankel and Toeplitz determinants for a subclass of $q$-starlike functions associated with a general conic domain. Mathematics 2019, 7, 1-15. [CrossRef]

22. Srivastava, H.M.; Altinkaya, S.; Yalcin, S. Hankel determinant for a subclass of bi-univalent functions defined by using a symmetric $q$-derivative operator. Filomat 2018, 32, 503-516. [CrossRef]

23. Zaprawa, P. Third Hankel determinants for subclasses of univalent functions. Med. J. Math. 2017. [CrossRef]

24. Zhang, H.-Y.; Tang, H.; Ma, L.-N. Upper bound of third Hankel determinant for a class of analytic functions. Pure Appl. Math. 2017, 33, 211-220. (In Chinese)

25. Zhang, H.-Y.; Tang, H.; Niu, X.-M. Third-order Hankel determinant for certain class of analytic functions related with exponential function. Symmetry 2018, 10, 1-8. [CrossRef]

26. Ali, M.F.; Thomas, D.K.; Vasudevarao, A. Toeplitz determinants whose elements are the coefficients of analytic and univalent functions. Bull. Aust. Math. Soc. 2018, 97, 253-264. [CrossRef]

27. Radhika, V.; Sivasubramanian, S.; Murugusundaramoorthy, G.; Jahangiri, J.M. Toeplitz matrices whose elements are the coefficients of functions with bounded boundary rotation. J. Complex Anal. 2016, 4960704, 4. [CrossRef]

28. Libera, R.J.; Zlotkiewicz, E.J. Coefficient bounds for the inverse of a function with derivative in P. Proc. Am. Math. Soc. 1983, 87, 251-257. [CrossRef]

29. Pommerenke, C. Univalent Functions; Vandenhoeck and Ruprecht: Gottingen, Germany, 1975. 